\title{
Efetividade dos aparelhos intrabucais de avanço mandibular no tratamento do ronco e da síndrome da apneia e hipopneia obstru- tiva do sono (SAHOS): revisão sistemática
}

Sergei Godeiro Fernandes Rabelo Caldas*, Alexandre Antonio Ribeiro**, Lourdes dos Santos-Pinto***,

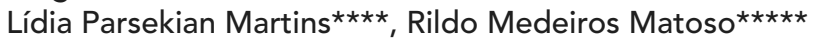

\section{Resumo}

Introdução: o ronco é produzido pela vibração do palato mole e/ou outros tecidos bucofaríngeos, durante a passagem do ar, na função respiratória. A SAHOS é definida como o fechamento de $30 \%$, no mínimo, da via aérea nasal/bucal por 10 segundos ou mais, apesar de existir esforço ventilatório, acompanhado de dessaturação da oxi-hemoglobina de 4\% ou mais. Objetivo: avaliar a evidência científica disponível sobre a utilização de aparelhos intrabucais de avanço mandibular no tratamento do ronco e da SAHOS. Métodos: uma estratégia de busca eletrônica utilizando palavras-chaves e critérios predefinidos foi realizada incluindo trabalhos publicados até outubro de 2008. Foi também utilizada a avaliação qualitativa da metodologia dos artigos. Resultados: embora um significativo número de estudos tenha analisado o assunto, apenas 7 artigos apresentaram qualidade metodológica para serem incluídos nesta revisão sistemática. Conclusões: os aparelhos intrabucais de avanço mandibular estão amplamente indicados para o tratamento do ronco e da SAHOS como terapia principal, ou alternativa naqueles pacientes que não podem suportar a terapia da pressão positiva das vias aéreas.

Palavras-chave: Transtornos do sono. Apneia obstrutiva do sono. Aparelhos ortodônticos. Avanço mandibular.

\section{INTRODUÇÃO}

O sono é uma função cerebral essencial à vida, como reparador contra o estresse humano. Os centros neurais que intervêm na produção e regulação do sono são localizados no tronco cerebral, diencéfalo e tálamo, com ampla expressão secun- dária do córtex. Hormônios, neurotransmissores e peptídeos ativos exercem uma forte modulação sobre os substratos neuroanatômicos que geram e modificam a fisiologia do sono ${ }^{5}$.

O sono humano normal é comprimido dentro de dois estágios distintos, conhecidos como sono

* Especialista em Ortodontia pela ABO/RN. Mestrando em Ortodontia pela Unesp/Araraquara.

** Especialista em Ortodontia pela ABO/RN. Mestrando em Ortodontia pela UFRJ.

*** Livre-docência em Odontopediatria pela Unesp/Araraquara. Professora adjunta do departamento de Clínica Infantil, disciplina de Odontopediatria, da Faculdade de Odontologia de Araraquara - Unesp.

**** Doutora em Ortodontia pela Unesp/Araraquara. Professora adjunta do departamento de Clínica Infantil, disciplina de Ortodontia, da Faculdade de Odontologia de Araraquara - Unesp.

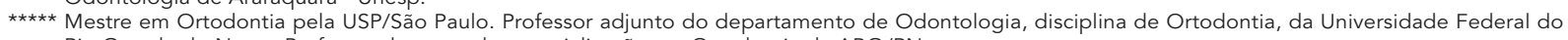
Rio Grande do Norte. Professor do curso de especialização em Ortodontia da ABO/RN. 
NREM (non rapid eyes movement $=$ movimento não rápido dos olhos) e sono REM (rapid eyes movement $=$ movimento rápido dos olhos). $\mathrm{O}$ ciclo de sono NREM-REM ocorre, aproximadamente, a cada 90 minutos - com quatro a seis ciclos por episódio de sono maior. O sono NREM é predominante no primeiro terço da noite e o sono REM é predominante no último terço ${ }^{5,15}$.

O padrão de sono é alterado durante o decorrer da vida. Recém-nascidos podem passar mais de 16 horas por dia dormindo, porém com sono intermitente e despertares nesse período. Em contrapartida, adultos apresentam um maior sono noturno, não acompanhado, na maioria dos casos, de cochilos durante o dia. Já as pessoas idosas possuem um padrão de sono fragmentado, onde grande parte do tempo é gasta na cama, porém com menos horas de sono ${ }^{5,15}$. Com o envelhecimento, os indivíduos ficam mais suscetíveis às desordens do sono, sendo as mais frequentes: o ronco e a síndrome da apneia e hipopneia obstrutiva do sono $(\mathrm{SAHOS})^{17}$.

O ronco é produzido pela vibração do palato mole e/ou outros tecidos bucofaríngeos durante a passagem do ar, na função respiratória. A incidência desse distúrbio é mais frequente entre os homens e é crescente até a sexta década de vida ${ }^{17}$.

A SAHOS é definida como o fechamento de $30 \%$, no mínimo, da via aérea nasal/bucal por 10 segundos ou mais, apesar de existir esforço ventilatório, acompanhado de dessaturação de $4 \%$ ou mais da oxi-hemoglobina. Sua prevalência aumenta com a idade até, aproximadamente, a sétima e oitava décadas de vida, sendo mais frequente entre os homens e em mulheres na menopausa. Existe, ainda, evidência científica do componente hereditário da $\mathrm{SAHOS}^{16}$.

Um decréscimo na amplitude respiratória entre 50 e $80 \%$, com duração superior a 10 segundos, é considerado uma hipopneia. Redução maior que $80 \%$, com duração maior que 10 segundos é considerada apneia ${ }^{17}$. Na apneia de origem central, uma interrupção total do fluxo aéreo pode ocorrer em virtude de alterações no sistema nervoso central e/ou periférico, impossibilitando a expansão da cavidade torácica e, consequentemente, o esforço inspiratório é nulo ${ }^{6,16}$.

Um importante critério de classificação e diagnóstico da SAHOS é o índice que reflete a frequência horária: índice de apneia (IA); índice de hipopneia (IH); índice de apneia e hipopneia (IAH). A apneia e a hipopneia obstrutiva do sono podem ser classificadas como leves, moderadas ou severas, com IAH de 5-15, 16-30 e > 30, respectivamente ${ }^{5,16,18}$.

Entre os fatores etiológicos do ronco e da SAHOS podemos citar o estreitamento anatômico das vias aéreas superiores (excesso de tecidos moles, macroglossia, micro e retrognatismo) predispondo, dessa maneira, a uma elevada resistência para o fluxo de ar, a qual gera uma pressão intraluminar negativa durante a inspiração, favorecendo a colapso respiratório ${ }^{5,6,9,12,16,18}$. O risco de se adquirir esses distúrbios aumenta significativamente com o ganho de peso, aumento da circunferência do pescoço e ingestão de bebidas alcoólicas. Condições sistêmicas também aparecem como fatores predisponentes, tais como: a hipertensão arterial sistêmica, hipotireoidismo não tratado, acromegalia e obstrução nasal ${ }^{16}$.

É conveniente lembrar que nem todo indivíduo que ronca possui a SAHOS, assim como nem todo portador da SAHOS, obrigatoriamente, ron$\mathrm{ca}$ - apesar desse ser um importante fator de diagnóstico ${ }^{4}$. Assim sendo, para se alcançar um diagnóstico preciso dessa síndrome, é fundamental a coleta de dados por meio da história clínica, do exame físico e realização de exames laboratoriais (polissonografia). Sono não-restaurador, sonolência diurna, fadiga, noctúria, alterações no desempenho cognitivo, irritabilidade, dores de cabeça matinais e movimentos anormais do corpo durante o sono são sinais e sintomas fundamentais para o diagnóstico diferencial da SAHOS. Em casos de dúvida, deve-se questionar os familiares dos portadores sobre os números de apneias e hipopneias 
por noite, se existem períodos de ronco - interrompidos por relativo silêncio e terminados pelo retorno do ronco - ou, ainda, reclamações do paciente sobre acordar ofegante ou com a sensação de choque ou sufocamento ${ }^{16,18}$.

Ainda que seja evidenciada clinicamente a presença da SAHOS, sua confirmação diagnóstica só é realizada através da polissonografia, com a complementação de dados adicionais: (1) parâmetros da qualidade, arquitetura e continuidade do sono; (2) fluxo de ar nasal/bucal; (3) qualidade e quantidade métrica do esforço respiratório; (4) saturação da oxi-hemoglobina pela oximetria de pulso; (5) eletrocardiograma; (6) posição do corpo para dormir, utilizando-se um sensor específico e monitor de vídeo ${ }^{16}$.

Existem diferentes modalidades de tratamento disponíveis para o ronco e a SAHOS, em função da enorme variabilidade de sinais e sintomas apresentados pelos pacientes. Há desde pacientes com ronco noturno primário, que procuram o tratamento apenas pelo ruído - que interfere no sono do(a) companheiro(a) -, até aqueles com apneia severa e sintomas acentuados de sonolência diurna, cansaço, fadiga e condições sistêmicas associadas ${ }^{6}$. Entre as modalidades de tratamento disponíveis podemos citar a redução do peso corpóreo, diminuição da ingestão de álcool, modificação da posição de dormir (tratamento comportamental), medicamentos, terapia da pressão positiva das vias aéreas, aparelhos intrabucais de avanço mandibular e reposicionamento lingual, cirurgia das vias aéreas superiores (tecidos moles) e ortognática (tecidos duros) ${ }^{16}$.

A efetividade dos tratamentos é dada por parâmetros laboratoriais (redução do IAH, redução dos microdespertares, melhora da dessaturação da oxihemoglobina e ausência de arritmia cardíaca) e parâmetros clínicos tais como a ausência de ronco ou redução significativa desse, melhora no grau de sonolência diurna excessiva e satisfação do paciente ${ }^{18}$.

Os aparelhos intrabucais de avanço mandibular previnem e minimizam o colapso das vias aéreas superiores durante o sono, pois promovem um aumento das vias aéreas, favorecendo, assim, uma maior passagem de ar. Dentre os efeitos adversos dessa terapia cita-se o desconforto na articulação temporomandibular e musculatura facial ${ }^{12}$, salivação ou secura excessiva da boca ${ }^{12}$, aumento do ângulo do plano mandibular ${ }^{1}$, diminuição de sobremordida e sobressaliência ${ }^{1}$, retroinclinação dos incisivos superiores ${ }^{1}$, proclinação dos incisivos inferiores, aumento da altura facial inferior, inclinação para distal dos molares superiores e inclinação mesial dos molares inferiores ${ }^{1}$. Essas alterações dentoesqueléticas são progressivas e resultantes de tratamentos muito longos, sendo necessário o acompanhamento com radiografias cefalométricas, modelos de estudos e fotografias intra e extrabucais, com o objetivo de minimizar esses efeitos.

O objetivo desse estudo foi realizar uma revisão sistemática sobre a efetividade dos aparelhos intrabucais de avanço mandibular no tratamento do ronco e da SAHOS.

\section{MATERIAL E MÉTODOS \\ Estratégia de busca}

Os artigos foram localizados por busca eletrônica semelhante à utilizada por Lim et al. ${ }^{12} \mathrm{e}$ desenvolvida para o MEDLINE (www.bireme. br) no período compreendido entre os anos de 1966 e 2008, sendo a busca mais recente realizada em outubro de 2008. Os termos utilizados para o levantamento bibliográfico foram "SLEEP" and ("APNOEA" or "APNEA" or "HYPOPNEA" or "HYPOPNOEA") or "SLEEP DISORDERED BREATHING" or "SLEEP RELATED RESPIRATORY DISORDER" or "SNORING" [Palavras] and oral or intraoral or intra-oral or intrabucal or intra-bucal or dental or tongue or mouth or jaw or mandib\$ or "mandib\$ advancement" or splint or prosth\$ or appliance or device [Palavras] and "ENSAIO CLINICO CONTROLADO" or "ENSAIO CONTROLADO ALEATORIO" [Tipo de publicação].

A complementação do levantamento foi reali- 
zada manualmente por meio da análise das referências bibliografias dos artigos de revisão pertinentes e de todos os ensaios clínicos controlados e aleatórios identificados.

\section{Critérios inicialmente escolhidos para a seleção dos estudos}

1) Ensaios clínicos controlados e aleatórios que utilizaram algum aparelho intrabucal de avanço mandibular no tratamento do ronco e da SAHOS.

2) Pacientes maiores de 16 anos de idade, de ambos os gêneros, com diagnóstico de ronco noturno primário e da SAHOS que apresentem cinco ou mais ocorrências de apneia e hipopneia por hora de sono.

3) Presença de grupo tratado (aparelho intrabucal de avanço mandibular) e grupo controle (aparelho intrabucal sem avanço mandibular).

4) Resultados primários que consideraram o número de ocorrências de apneia e hipopneia por horas de sono e a sonolência diurna, e as variáveis secundárias avaliadas foram: índices de dessaturação da oxi-hemoglobina, frequência e altura do ronco, qualidade de vida, função cognitiva, efeitos adversos da terapia e preferência do paciente.

\section{Seleção dos estudos}

Dois autores examinaram, de forma independente, os títulos, as palavras-chaves e os resumos dos relatos identificados no banco de dados, para evidenciação de três critérios:

- Ensaios clínicos controlados e aleatórios.

- Utilização de algum dispositivo intrabucal de avanço mandibular em pacientes com diagnóstico de ronco noturno primário e da SAHOS.

- Grupo controle com as mesmas características do grupo tratado, recebendo um aparelho intrabucal sem avanço mandibular.

Os artigos que preencheram os critérios de seleção, descritos nos resumos, foram selecionados. Estudos com resumos que não continham informações necessárias para o julgamento e estudos com títulos interessantes que não apresentavam resumos no banco eletrônico de dados foram adquiridos na íntegra. Os casos com julgamentos não-concordantes foram solucionados mediante consenso.

A avaliação qualitativa da metodologia aplicada aos estudos foi realizada de acordo com os trabalhos descritos por Antczak, Tang, Chalmers² e Jadad et al. ${ }^{10}$ As variáveis levadas em consideração foram: tipo do estudo (estudo controlado e aleatório $=3$ pontos; estudo prospectivo $=1$ ponto; estudo retrospectivo $=0$ ); tamanho da amostra $\geq 30=$ 1 ponto; método correto de utilização da amostra = 1 ponto; coerência da metodologia empregada = 1 ponto; utilização da análise de erro do método $=1$ ponto; cegamento das medidas $=1$ ponto; testes estatísticos adequados $=1$ ponto; fatores de confundimento $=1$ ponto. Cada artigo, portanto, poderia atingir a pontuação máxima de 10 pontos, sendo posteriormente classificado segundo a qualidade do trabalho (baixa $=0-5$ pontos; média $=$ 6-8 pontos; e alta $=9-10$ pontos) (Tab. 1$)$.

\section{RESULTADOS}

Inicialmente, foram classificados e identificados 185 resumos que utilizaram algum dispositivo de avanço mandibular no tratamento do ronco e da SAHOS. Desses, apenas sete foram incluídos na revisão, de acordo com os critérios preestabelecidos, visando maior grau de evidência científica (Tab. 2).

\section{DISCUSSÃO}

A Sociedade Brasileira de Sono, Rinologia e Otorrinolaringologia, em 2000, propôs um protocolo clínico para o diagnóstico e intervenção terapêutica do tratamento do ronco e da SAHOS. Inicialmente, o médico responsável deve realizar uma avaliação clínica e o preenchimento de questionários sobre a história do sono e qualidade de vida do paciente. Em casos de suspeita clínica da SAHOS, esse deve ser submetido a uma polissonografia, para se alcançar um diagnóstico definitivo da síndrome ${ }^{18}$. 
TABELA 1 - Avaliação qualitativa dos estudos selecionados.

\begin{tabular}{|c|c|c|c|c|c|c|c|c|c|}
\hline $\begin{array}{l}\text { autor } \\
\text { (ano) }\end{array}$ & $\begin{array}{l}\text { tipo do } \\
\text { estudo }\end{array}$ & $\begin{array}{l}\text { tamanho da } \\
\text { amostra }\end{array}$ & $\begin{array}{l}\text { método } \\
\text { correto de } \\
\text { utilização da } \\
\text { amostra }\end{array}$ & $\begin{array}{c}\text { coerência da } \\
\text { metodologia } \\
\text { empregada }\end{array}$ & $\begin{array}{l}\text { utilização } \\
\text { da análise } \\
\text { de erro do } \\
\text { método }\end{array}$ & $\begin{array}{l}\text { cega- } \\
\text { mento } \\
\text { das medi- } \\
\text { das }\end{array}$ & $\begin{array}{l}\text { testes } \\
\text { estatísticos } \\
\text { adequados }\end{array}$ & $\begin{array}{l}\text { fatores de } \\
\text { confundi- } \\
\text { mento }\end{array}$ & $\begin{array}{c}\text { padrão de } \\
\text { qualidade } \\
\text { conside- } \\
\text { rado }\end{array}$ \\
\hline $\begin{array}{l}\text { Hans et al. }{ }^{8} \\
\text { (1997) }\end{array}$ & $\begin{array}{c}\text { estudo } \\
\text { controlado } \\
\text { e aleatório }\end{array}$ & inadequado & adequado & adequado & não & $\operatorname{sim}$ & $\operatorname{sim}$ & não & alto \\
\hline $\begin{array}{l}\text { Mehta et al. }{ }^{13} \\
\text { (2001) }\end{array}$ & $\begin{array}{c}\text { estudo } \\
\text { controlado } \\
\text { e aleatório }\end{array}$ & inadequado & adequado & adequado & não & $\operatorname{sim}$ & $\operatorname{sim}$ & não & alto \\
\hline $\begin{array}{l}\text { Johnston et al. }{ }^{11} \\
\qquad(2002)\end{array}$ & $\begin{array}{c}\text { estudo } \\
\text { controlado } \\
\text { e aleatório }\end{array}$ & inadequado & adequado & adequado & não & $\operatorname{sim}$ & $\operatorname{sim}$ & não & alto \\
\hline $\begin{array}{l}\text { Gotsopoulos et al. }{ }^{7} \\
(2002)\end{array}$ & $\begin{array}{c}\text { estudo } \\
\text { controlado } \\
\text { e aleatório }\end{array}$ & adequado & adequado & adequado & não & $\operatorname{sim}$ & $\operatorname{sim}$ & não & alto \\
\hline $\begin{array}{c}\text { Blanco et al. }{ }^{3} \\
(2005)\end{array}$ & $\begin{array}{c}\text { estudo } \\
\text { controlado } \\
\text { e aleatório }\end{array}$ & inadequado & adequado & adequado & não & $\operatorname{sim}$ & $\operatorname{sim}$ & não & alto \\
\hline $\begin{array}{c}\text { Cooke, Battagel }{ }^{4} \\
\text { (2006) }\end{array}$ & $\begin{array}{c}\text { estudo } \\
\text { controlado } \\
\text { e aleatório }\end{array}$ & inadequado & adequado & adequado & $\operatorname{sim}$ & $\operatorname{sim}$ & $\operatorname{sim}$ & não & alto \\
\hline $\begin{array}{l}\text { Petri et al. }{ }^{14} \\
(2008)\end{array}$ & $\begin{array}{c}\text { estudo } \\
\text { controlado } \\
\text { e aleatório }\end{array}$ & adequado & adequado & adequado & $\operatorname{sim}$ & $\operatorname{sim}$ & $\operatorname{sim}$ & não & alto \\
\hline
\end{tabular}

O padrão-ouro para o tratamento da SAHOS é a terapia da pressão positiva das vias aéreas CPAP (continuos positive airway pressure $=$ pressão positiva e contínua nas vias aéreas superiores), contudo a colaboração dos pacientes na utilização desse mecanismo ainda é um problema ${ }^{11}$. Entre as principais causas da não-adesão a esse tratamento estão a claustrofobia e o desconforto facial causados pela máscara ${ }^{18}$.

Nesse contexto, os aparelhos intrabucais de avanço mandibular vêm ganhando cada vez mais espaço no tratamento do ronco e da SAHOS. Esses dispositivos possuem baixo custo de confecção, são bem aceitos pela maioria dos pacientes e os possíveis efeitos adversos, como o desconforto muscular e temporomandibular, são transitórios ${ }^{11}$. Em contrapartida, em tratamentos muito longos, como geralmente ocorre, os efeitos adversos dentários e esqueléticos da terapia são progressivos, sendo, dessa maneira, fundamental que o tratamento seja conduzido por um ortodontista capacitado, para poder minimizá-los. Durante as décadas passadas, diversos trabalhos reportaram a efetividade desse tratamento no ronco e na SAHOS, porém eram relatos de casos clínicos ou de uma série de casos ${ }^{9}$.

Na presente revisão sistemática da literatura, sobre a efetividade dos aparelhos intrabucais no manejo dessas desordens do sono, apenas sete ensaios clínicos controlados e aleatórios preencheram o grau de evidência científica adotado como critério de seleção.

Dos sete estudos selecionados, seis avaliaram a ação dos aparelhos intrabucais no tratamento da SAHOS $^{3,7,8,11,13,14}$ e um no tratamento do ronco não-apneico ${ }^{4}$.

Os resultados dos trabalhos foram divididos dentro de parâmetros subjetivos (descrição dos sintomas e preenchimento de questionários) e objetivos (resultados polissonográficos). O dado subjetivo mais reportado foi a escala de sonolência 
TABELA 2 - Descrição dos artigos selecionados para revisão sistemática.

\begin{tabular}{|c|c|c|c|c|}
\hline $\begin{array}{l}\text { autor } \\
\text { (ano) }\end{array}$ & revista & amostra & $\begin{array}{c}\text { avanço } \\
\text { mandibular }\end{array}$ & variáveis avaliadas \\
\hline $\begin{array}{c}\text { Hans et al. }{ }^{8} \\
\text { (1997) }\end{array}$ & $\begin{array}{l}\text { Am. J. } \\
\text { Orthod. } \\
\text { Dentofacial } \\
\text { Orthop. }\end{array}$ & $\begin{array}{l}24 \text { indivíduos voluntários foram selecionados, sendo que } \\
18 \text { completaram o protocolo. } \\
\text { Média de idade: } 51,9 \pm 12,3 \text { anos }\end{array}$ & 6 a $8 \mathrm{~mm}$ & IDR e ESE \\
\hline $\begin{array}{l}\text { Mehta et al. }{ }^{13} \\
\text { (2001) }\end{array}$ & $\begin{array}{l}\text { Am. J. Res- } \\
\text { pir. Crit. Care } \\
\text { Med. }\end{array}$ & $\begin{array}{l}28 \text { participantes ( } 22 \text { homens e } 6 \text { mulheres) foram selecio- } \\
\text { nados, sendo que } 24 \text { ( } 19 \text { homens e } 5 \text { mulheres) completa- } \\
\text { ram o protocolo. Média de idade: } 48 \pm 9 \text { anos; } \\
\text { IMC: } 29,4 \pm 3,1 \mathrm{~kg} / \mathrm{m}^{2} \text {; IAH: } 27 \pm 17 / \mathrm{h} \text {; } \mathrm{MinSaO}_{2}: 85 \pm 8 \%\end{array}$ & $7,5 \pm 1,8 \mathrm{~mm}$ & $\begin{array}{l}\text { Frequência do ronco, intensida- } \\
\text { de do ronco, ESE, IAH, MinSaO }{ }^{\prime} \\
\text { índice de despertares e satisfa- } \\
\text { ção com o tratamento }\end{array}$ \\
\hline $\begin{array}{l}\text { Johnston et al. }{ }^{11} \\
\qquad(2002)\end{array}$ & $\begin{array}{l}\text { Eur. J. } \\
\text { Orthod. }\end{array}$ & $\begin{array}{l}21 \text { pacientes (17 homens e } 4 \text { mulheres) foram seleciona- } \\
\text { dos, sendo que } 20 \text { pacientes ( } 16 \text { homens e } 4 \text { mulheres) } \\
\text { completaram o protocolo. Média de idade: } 55,10 \pm 6,87 \\
\text { anos; IAH: } 31,93 \pm 21,18 / h \text {; ESE: } 13,90 \pm 6,9 ; \text { dessaturação } \\
\text { do oxigênio arterial: } 30,69 \pm 18,82 \% \text {; IMC: } 31,63 \pm 5,94 \mathrm{~kg} / \mathrm{m}^{2}\end{array}$ & 4 a $9 \mathrm{~mm}$ & $\begin{array}{l}\text { IAH, dessaturação do oxigênio } \\
\text { arterial, frequência e } \\
\text { altura do ronco, ESE, } \\
\text { tolerabilidade e cooperação }\end{array}$ \\
\hline $\begin{array}{l}\text { Gotsopoulos et al. }{ }^{7} \\
(2002)\end{array}$ & $\begin{array}{l}\text { Am. J. Res- } \\
\text { pir. Crit. Care } \\
\text { Med. }\end{array}$ & $\begin{array}{l}85 \text { participantes foram selecionados, sendo que } 73 \\
\text { participantes ( } 59 \text { homens e } 14 \text { mulheres) completaram } \\
\text { o protocolo. Média de idade: } 48 \pm 11 \text { anos; IAH: } 27 \pm 2 / \mathrm{h} \text {; } \\
\qquad \text { MinSaO }_{2}: 86 \pm 1 \% \text {; ESE: } 11\end{array}$ & $7,2 \pm 2 \mathrm{~mm}$ & $\begin{array}{l}\text { IDR, ESE, } \mathrm{MinSaO}_{2} \text {, frequência } \\
\text { e intensidade do ronco, teste } \\
\text { de latência múltipla do sono e } \\
\text { satisfação do tratamento }\end{array}$ \\
\hline $\begin{array}{l}\text { Blanco et al. }{ }^{3} \\
(2005)\end{array}$ & Sleep Breath & $\begin{array}{l}24 \text { participantes ( } 20 \text { homens e } 4 \text { mulheres) foram selecio- } \\
\text { nados, sendo que } 15 \text { pacientes ( } 13 \text { homens e } 2 \text { mulheres) } \\
\text { completaram o protocolo. Média de idade: } 55 \text { anos; } \\
\text { IMC: } 26,8 \mathrm{~kg} / \mathrm{m}^{2} \text {, IAH: } 24-33 / \mathrm{h} \text {; ESE: } 14,7-16,3\end{array}$ & $\begin{array}{l}75 \% \text { do } \\
\text { avanço } \\
\text { máximo } \\
\text { permitido }\end{array}$ & $\begin{array}{l}\text { IAH, número de despertares no- } \\
\text { turnos, frequência e intensidade } \\
\text { do ronco, ESE, qualidade de vida } \\
\text { e saúde, e satisfação } \\
\text { do tratamento }\end{array}$ \\
\hline $\begin{array}{l}\text { Cooke e Battagel } \\
\qquad(2006)\end{array}$ & $\begin{array}{l}\text { Eur. J. } \\
\text { Orthod. }\end{array}$ & $\begin{array}{l}27 \text { indivíduos ( } 17 \text { homens e } 10 \text { mulheres) foram selecio- } \\
\text { nados, sendo que } 23 \text { completaram o protocolo. Média de } \\
\text { idade: } 44,7 \text { anos (variando de } 29,2 \text { a } 63,5 \text { anos); } \\
\text { IMC: } 27,1 \mathrm{~kg} / \mathrm{m}^{2} \text { (variando de } 20,3 \text { a } 35,1 \mathrm{~kg} / \mathrm{m}^{2} \text { ) }\end{array}$ & $\begin{array}{l}75 \% \text { do } \\
\text { avanço } \\
\text { máximo } \\
\text { permitido }\end{array}$ & $\begin{array}{l}\text { Questionários sobre a história } \\
\text { do sono, ESE, altura do ronco, } \\
\text { qualidade de vida dos portado- } \\
\text { res do ronco, qualidade de vida } \\
\text { dos seus parceiros, frequência } \\
\text { do ronco, IAH, } \mathrm{SaO}_{2} \text {, parâmetros } \\
\text { cefalométricos e tolerabilidade }\end{array}$ \\
\hline $\begin{array}{l}\text { Petri et al. }{ }^{14} \\
\text { (2008) }\end{array}$ & J. Sleep Res. & $\begin{array}{l}93 \text { pacientes ( } 76 \text { homens e } 17 \text { mulheres) foram sele- } \\
\text { cionados, sendo que } 81 \text { participantes ( } 66 \text { homens e } 15 \\
\text { mulheres) completaram o protocolo. Média de idade: } \\
50 \pm 11 \text { (grupo tratado), } 50 \pm 10 \text { (grupo controle), } 49 \pm 10 \\
\text { (grupo controle sem intervenção); IMC: } 31,3 \mathrm{~kg} / \mathrm{m}^{2} \text { (varian- } \\
\text { do de } 30,1 \text { a } 32,6 \text { ), IAH: } 34,7 / \mathrm{h} \text { (variando de } 29,7 \text { a } 39,6 \text { ); } \\
\text { ESE: } 11 \text { (variando de } 10 \text { a } 11,9 \text { ) }\end{array}$ & $\begin{array}{l}74 \% \text { do } \\
\text { avanço } \\
\text { máximo } \\
\text { permitido }\end{array}$ & IAH, ESE e qualidade de vida \\
\hline
\end{tabular}

IAH: índice de apneia e hipopneia; IDR: índice de distúrbios respiratórios; IMC: índice de massa corpórea; ESE: escala de sonolência Epworth; $\mathrm{MinSaO}_{2}$ : saturação mínima do oxigênio arterial; $\mathrm{SaO}_{2}$ : saturação do oxigênio arterial.

Epworth (ESE), enquanto os dados objetivos foram o IAH e a dessaturação da oxi-hemoglobina.

A escala de sonolência Epworth (ESE) foi desenvolvida para mensurar a sonolência diurna em pessoas com suspeita de desordens do sono. Os valores da ESE variam de 0 a 24 , com os escores mais baixos indicando redução da sonolência diurna e os escores elevados indicando aumento da sonolência diurna. Em indivíduos com hábitos normais de sono, sem apneia, a ESE é de 06, enquanto em portadores da SAHOS moderada a severa os escores variam de 10 a $16^{8}$. Em todos os estudos selecionados os autores reportaram uma redução nos escores da $\mathrm{ESE}^{3,4,7,8,13,14}$ em pacientes portadores do ronco primário e SAHOS, exceto no estudo de Johnston et al. ${ }^{11}$, no qual essa melhora não foi estatisticamente significativa. Uma possível explicação desse resultado é que a sonolência diurna pode ter outros fatores contribuintes além de, simplesmente, a SAHOS ${ }^{11}$.

$\mathrm{O}$ índice de ocorrência de apneia e hipopneia (IAH) reflete a frequência horária desses eventos, 
sendo um importante meio de classificação e diagnóstico da SAHOS. A apneia e a hipopneia obstrutiva do sono podem ser classificadas como leve, moderada ou severa, com IAH de 5-15, 16-30 e $>30$, respectivamente ${ }^{5,16,18}$. Alguns estudos descrevem o IAH como sendo um índice de distúrbios respiratórios (IDR), porém ambos possuem o mesmo significado clínico ${ }^{7,8}$.

Avaliando os resultados dos trabalhos selecionados, os pacientes que utilizaram o aparelho intrabucal de avanço mandibular tiveram uma melhora estatisticamente significativa na frequência horária do $\mathrm{IAH}^{7,8,11,13,14}$. Porém, Blanco et al. ${ }^{3}$ observaram que o grupo tratado com o aparelho intrabucal de avanço mandibular apresentou uma redução maior no IAH e mais da metade dos pacientes relatou um controle total dos sintomas da SAHOS, bem como uma melhora na qualidade de vida, comparados aos pacientes do grupo controle - que usaram aparelho similar sem avanço mandibular. Em contrapartida, Cooke e Battagel ${ }^{4}$ reportaram que o IAH não apresentou melhora significativa nos grupos avaliados. No presente estudo, os indivíduos eram portadores de ronco primário não-apneico e IAH próximo do normal (pouco maior que 5) na linha base, fato provavelmente responsável por não haver diferença significativa nos escores do IAH após a utilização do TheraSnore $^{\mathrm{TM}}$ (Distar UK Ltd, Reino Unido) com e sem avanço mandibular.

Um dos sintomas mais prevalentes e graves da SAHOS é a dessaturação da oxi-hemoglobina. A saturação mínima do oxigênio diminui muito durante os episódios de apneia e hipopneia e mantém-se abaixo dos índices normais (90\%) durante toda a noite. Para compensar a dessaturação da oxi-hemoglobina (redução do $\mathrm{O}_{2}$ arterial e elevação do $\mathrm{CO}_{2}$ arterial), ocorre um aumento no trabalho cardíaco, especificamente no ventrículo direito, para que possa chegar sangue aos pulmões e ocorram as trocas gasosas $\left(\mathrm{CO}_{2}\right.$ pelo $\mathrm{O}_{2}$ ), a fim de restabelecer os níveis adequados de oxigênio arterial para que os tecidos nobres sejam adequadamente oxigenados. Esse aumento na atividade do coração, especificamente no ventrículo direito, de forma crônica (pacientes com SAHOS não-tratados) pode ocasionar uma condição patológica denominada Cor Pulmonale. Não havendo tratamento para a correção da obstrução das vias aéreas e, consequentemente, normalização da saturação da oxi-hemoglobina, o coração poderá ir à falência, levando o paciente a óbito ${ }^{17}$.

Os estudos que avaliaram a dessaturação da oxi-hemoglobina apresentaram resultados bastante satisfatórios ${ }^{7,11,13}$. A avaliação desse índice pode ser realizada pela saturação mínima do oxigênio arterial $\left(\mathrm{MinSaO}_{2}\right)$, saturação do oxigênio $\left(\mathrm{SaO}_{2}\right)$ ou pela sua dessaturação. Os pacientes que utilizaram os dispositivos de avanço mandibular obtiveram uma melhora estatisticamente significativa nas $\mathrm{MinSaO}_{2}$ e $\mathrm{SaO}_{2}$ (aumento da porcentagem $)^{4,7,13}$, assim como aqueles estudos que avaliaram a dessaturação do oxigênio obtiveram índices menores (diminuição da porcentagem) ${ }^{11}$ ao final da terapia. Contudo, ambos os resultados significaram a elevação do oxigênio arterial e melhora na função cardiopulmonar. Apenas Cooke e Battagel $^{4}$ demonstraram que esse índice não apresentou melhora significativa nos grupos avaliados.

O ronco noturno é caracterizado por um ruído de intensidade e frequência variada, sendo bastante incômodo para os parceiros de seus portadores. Como foi citado anteriormente, o ronco pode ocorrer sem que o paciente apresente a SAHOS, assim como pode estar ausente nos portadores dessa síndrome ${ }^{4}$. Avaliando os resultados dos estudos selecionados, pode-se constatar que o avanço mandibular constitui-se como uma alternativa bastante viável para redução da frequência e intensidade do ronco ${ }^{3,4,7,13}$. Porém, no estudo de Johnston et al. ${ }^{11}$, essa melhora foi estatisticamente não significativa. Apesar de os resultados terem sidos significativos, com relação à melhora do ronco, nos estudos em que os participantes eram portadores da SAHOS $3,7,13$, esses pacientes apresentaram alterações mais pronunciadas na forma e função das 
vias aéreas superiores em comparação com os pacientes que possuíam apenas o ronco não-apneico. Dessa maneira, o avanço mandibular é bastante efetivo para os roncadores primários e pode constituir uma alternativa viável no tratamento desse sintoma nos portadores da SAHOS, contudo os resultados poderão não ser tão previsíveis ${ }^{11}$.

Em relação à qualidade do sono (redução dos despertares noturno), houve uma melhora estatisticamente significativa naqueles indivíduos que utilizaram o aparelho de avanço mandibular ${ }^{3,7,13}$. No entanto, Cooke e Battagel ${ }^{4}$ observaram que essa melhora foi não significativa, se comparada à linha base e nem entre os grupos, contudo os parceiros dos pacientes descreveram uma melhora na qualidade do próprio sono quando esses utilizavam o aparelho ativo.

Quando avaliadas a tolerabilidade e colaboração com o tratamento, os pacientes reportaram: poucos efeitos colaterais, grande tempo de uso do aparelho, desejo de continuar utilizando-o e satisfação com o tratamento, em virtude da melhora perceptível dos sintomas $3,4,7,11,13,14$.

\section{CONCLUSÕES}

Após a avaliação dos trabalhos selecionados para a revisão sistemática, pode-se afirmar que os aparelhos de avanço mandibular promoveram:

- Redução da sonolência diurna.

- Redução da frequência horária dos episódios de apneia e hipopneia.

- Elevação da concentração do oxigênio arterial.

- Redução na frequência e intensidade do ronco, sendo mais significativo em pacientes com ronco primário não associado à SAHOS.

- Melhora na qualidade do sono dos portadores do ronco e da SAHOS, assim como de seus parceiros.

- Boa tolerabilidade em virtude, principalmente, da redução dos sintomas.

The effectiveness of mandibular advancement intraoral appliances in the treatment of the snoring and obstructive sleep apnea and hypopnea syndrome (OSAHS): systematic review

\begin{abstract}
Introduction: The snoring is produced by the vibration of the soft palatum and/or other oropharyngeal tissues, during the air passage, in the breathing function. OSAHS is defined as the closing of $30 \%$, at least, of the nasal/ buccal airway for 10 seconds or more, in spite of existing inspiratory effort, accompanied by oxyhemoglobin desaturation of $4 \%$ or more. Objectives: To evaluate the available scientific evidence about the use of mandibular advancement intraoral appliances in the treatment of the snoring and/or OSAHS. Methodology: Electronic search strategy using predefined key-words and criteria was realized including studies published until October of 2008. It was also used the qualitative evaluation of the articles methodology. Results: Although a significant number of articles has studied this subject, only 7 articles showed methodological quality to be included in this systematic review. Conclusions: The intraoral appliances are widely prescribed for the treatment of snoring and OSAHS as primary therapy and as an alternative to patients who are unable to tolerate the positive airway pressure therapy.
\end{abstract}

Keywords: Sleep disorders. Obstructive sleep apnea. Orthodontic appliances. Mandibular advancement. 


\section{REFERÊNCIAS}

1. ALMEIDA, F. R.; LOWE, A. A.; SUNG, J. O.; TSUIKI, S.; OTSUKA, R. Long-term sequelae of oral appliance therapy in obstructive sleep apnea patients: Part 1. Cephalometric analysis. Am. J. Orthod. Dentofacial Orthop., St. Louis, v. 129, no. 2, p. 195-204, Feb. 2006.

2. ANTCZAK, A. A.: TANG, J.: CHALMERS, T. C. Quality assessment of randomized control trials in dental research. I. Methods. J. Periodontal Res., Copenhagen, v. 21, no. 4, p. 305-314, July 1986

3. BLANCO, J.; ZAMARRÓN, C.; ABELEIRA PAZOS, M. T.; LAMELA, C.; SUAREZ QUINTANILLA, D. Prospective evaluation of an oral appliance in the treatment of obstructive sleep apnea syndrome. Sleep Breath, Heidelberg, v. 9, no. 1, p. 20-25, Mar. 2005.

4. COOKE, M. E.; BATTAGEL, J. M. A thermoplastic mandibular advancement device for the management of non-apnoeic snoring: a randomized controlled trial. Eur. J. Orthod., Oxford, v. 28, no. 4, p. 327-338, Aug. 2006.

5. CULEBRAS, A. Normal sleep. In: LEE-CHIONG Jr., T. L.; SATEIA, M. J.; CARSKADON, M. A. (Ed.). Sleep Medicine. Philadelphia: Hanley \& Belfus, 2002. p. 151-160.

6. FANTINI, S. M. Tratamento da síndrome da apnéia do sono obstrutiva e ronco noturno e sua influência no tratamento ortodôntico. In: CARDOSO, R. J. A.; GONCCALVES, E. A. N. (Ed.). Ortodontia e Ortopedia Funcional dos Maxilares. São Paulo: Artes Médicas, 2002. v. 7, p. 185-199.

7. GOTSOPOULOS, H.; CHEN, C.; OIAN, J.; CISTULLI, P. A. Oral appliance therapy improves symptoms in obstructive sleep apnea: a randomized, controlled trial. Am. J. Respir. Crit. Care Med., New York, v. 166, no. 5, p. 743-748, Sept. 2002

8. HANS, M. G.; NELSON, S.; LUKS, V. G.; LORKOVICH, P.; BAEK, S. J. Comparison of two dental devices for treatment of obstructive sleep apnea syndrome (OSAS). Am. J. Orthod. Dentofacial Orthop., St. Louis, v. 111, no. 5, p. 562-570, May 1997.

9. HOFFSTEIN, V. Review of oral appliances for treatment of sleep-disordered breathing. Sleep Breath, Heidelberg, v. 11, no. 1, p. 1-22, Mar. 2007.

10. JADAD, A. R.; MOORE, R. A.; CARROLL, D.; JENKINSON, C.; REYNOLDS, D. J.; GAVAGHAN, D. J.; McQUAY, H. J. Assessing the quality of reports of randomized clinical trials: is blinding necessary? Control Clin. Trials, New York, v. 17, no. 1, p. 1-12, Feb. 1996.
11. JOHNSTON, C. D.; GLEADHILL, I. C.; CINNAMOND, M. J.; GABBEY, J.; BURDEN, D. J. Mandibular advancement appliances and obstructive sleep apnoea: a randomized clinical trial. Eur. J. Orthod., Oxford, v. 24, no. 3, p. 251-262, June 2002.

12. LIM, J.; LASSERSON, T. J.; FLEETHAM, J.; WRIGHT, J. Oral appliances for obstructive sleep apnoea. Cochrane Database Syst. Rev., no. 1, 2006. DOI: 10.1002/14651858.CD004435. pub3. Disponível em: <http://mrw.interscience.wiley.com/ cochrane/clsysrev/articles/CD004435/frame.html>. Acesso em: 28 jan. 2008

13. MEHTA, A. J.; QIAN, P.; PETOCZ, M. A.; DARENDELILER, P. A. CISTULLI, P. A. A randomized, controlled study of a mandibular advancement splint for obstructive sleep apnea. Am. J. Respir. Crit. Care Med., New York, v. 163, no. 6, p. 1457-1461, May 2001.

14. PETRI, N.; SVANHOLT, P.; SOLOW, B.; WILDSCHIODTZ, G.; WINKEL, P. Mandibular advancement appliance for obstructive sleep apnoea: results of a randomized placebo controlled trial using parallel group design. J. Sleep. Res., Oxford, v. 17, no. 2, p. 221-229, June 2008

15. RAMA, A. N.; CHO, S. C.; KUSHIDA, C. A. Normal human sleep. In: LEE-CHIONG, T. (Ed.). Sleep: a comprehensive handbook. New Jersey: John Wiley, 2006. p. 3-9.

16. SANDERS, M. H.; GIVELBER, R. J. Overview of obstructive sleep apnea in adults. In: LEE-CHIONG, T. (Ed.). Sleep: a comprehensive handbook. New Jersey: John Wiley, 2006. p. 231-239.

17. SIMÕES, D. O. et al. O papel do ortodontista na síndrome da apnéia do sono obstrutiva (SASO) e a cefalometria específica. In: ALVES, F. A. (Ed.). Ortodontia: terapia biofuncional. São Paulo: Ed. Santos, 2003. p. 255-310.

18. TUFIK, S.; SAMPAIO, P. L.; WECKX, L. L. M. et al. Consenso em ronco e apnéia do sono. São Paulo: Sociedade Brasileira de Sono, Rinologia e Otorrinolaringologia, 2000.

\section{Endereço para correspondência}

Sergei Godeiro Fernandes Rabelo Caldas

Rua Humaitá - 1740, apto. 36 - Centro

CEP: 14.801-385 - Araraquara / SP

E-mail: sergeirabelo@uol.com.br 